\title{
Case Reports - Special Supplement
}

The first special supplement of case reports of the year 2019 is presented.

The Journal of College of Physicians and Surgeons Pakistan is continuously receiving a large number of case reports of clinical importance for publication, which has necessitated an increase in the frequency of case report supplements.

We hope that the medical professionals would benefit from this compilation of interesting case reports from multiple disciplines. 